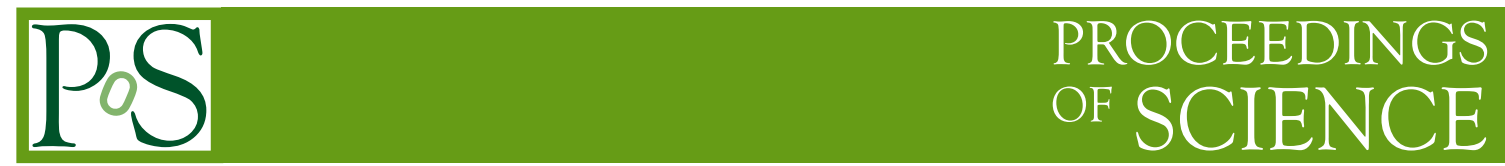

\title{
The Very High Energy $\gamma$-ray Extragalactic Sky
}

\author{
Arache Djannati-Atai* \\ Arache Djannati-Atai \\ E-mail: djannati@in2p3.fr
}

\begin{abstract}
After a decade of operation of third generation Imaging Atmospheric Cherenkov telescopes, not only the number and our knowledge of very high energy $\gamma$-ray $(>\sim 100 \mathrm{GeV})$ extragalactic sources have increased significantly, the use of their emission, either as probes of the intergalactic medium for cosmological applications, or as tools for exploring frontiers of fundamental physics, has revealed itself a very essential tool. I will review the field and discuss the latest results from major observatories.
\end{abstract}

Frontiers of Fundamental Physics 14 - FFP14,

15-18 July 2014

Aix Marseille University (AMU) Saint-Charles Campus, Marseille

* Speaker. 